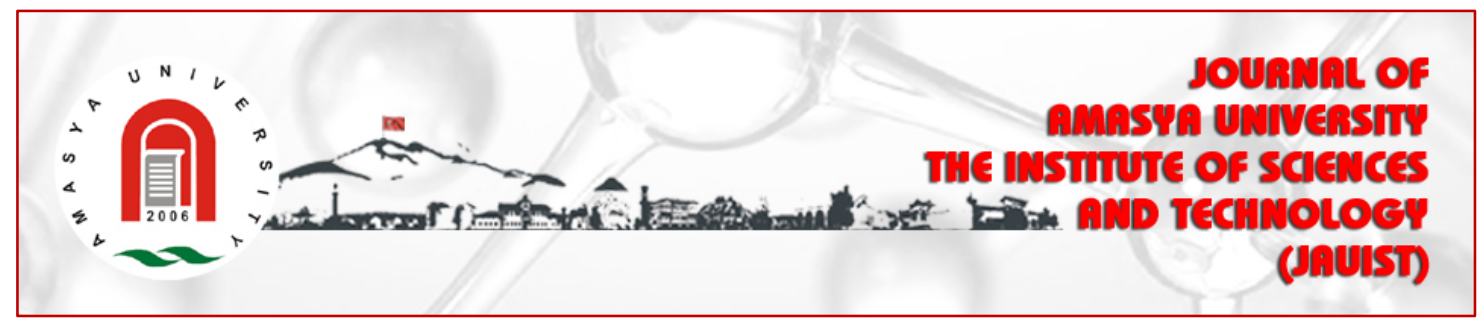

\title{
SOME PHYSICAL PROPERTIES OF HALF-HEUSLER COMPOUND NAYSI : FIRST-PRINCIPLES STUDY
}

\author{
${ }^{* 1}$ Yasemin Oztekin Ciftci
}

${ }^{*}$ Gazi University, Department of Physics, Teknikokullar, 06500 Ankara, Turkey ORCID*1: ID/0000-0003-1796-0270

Research Type: Original Research Article

Received: 20.12.2021, Accepted: 30.12.2021

*Corresponding author: yasemin@gazi.edu.tr

https://doi.org/10.54559/jauist.1039136

\section{Abstract}

The structural, electronic, elastic and optic properties of NaYSi formed in the half-Heusler structure are studied by using ab-initio density-functional theory. The calculated equilibrium lattice constants are compaired with the available experimental and theoretical data. The elastic parameters have calculated. Computed elastic results prove that this compound were mechanically stable. The band gap of this compound predicted to be semiconductor. Further the phonon spectra and optical analysis have been obtained for the energy range of 0-40 eV.

Key Words: Half-Heusler, electronic, elastic, optic. 


\section{Özet}

Yarı-Heusler yapısındaki NaYSi bileşiğinin yapısal, elektronik, elastik ve optik özellikleri abinitio yoğunluk-fonksiyonel teori kullanılarak incelenmiştir. Hesaplanan denge örgü sabitleri, mevcut deneysel ve teorik verilerle karşılaştırılmıştır. Elastik parametreler hesaplanmıştır. Elastik sonuçlarımız, bu bileşiğin mekanik olarak kararlı olduğunu kanıtlıyor. Bu bileşiğin yarı iletken olduğu bant aralığından tahmin edilmiştir. Ayrıca, 0-40 eV enerji aralı̆̆ı için fonon spektrumları ve optik analiz yapılmıştır.

Anahtar Kelimeler: Yarı-Heusler, elektronik, elastik, optik.

\section{Introduction}

Half-Heusler compounds (space group F43m, 216) contain a relatively large family of materials with different physical properties and applications. Topological insulators, piezoelectric semiconductors, thermoelectric semiconductors and optoelectronic semiconductors are among the functional materials based on these compounds.

Some binary semiconductors have limited applications in various fields. However halfHeusler compounds with chemical composition XYZ or "Nowotny-Juza" belong to a special class of materials [1]. Many semiconductors with wide band gaps are in the class of eight-electron half-Heusler compounds [2]. So far, little work has been done with ab initio calculations [3-9] on possible eight-electron quasi-Heusler compounds of types I-II-V and I-III-IV.

Hem yarı Heusler bileşikleri hem de ikili yarı iletkenler için elde edilen bazı fiziksel özellikler, yeni yarı Heusler yarı iletkenleri bulmamıza yardımcı olmaktadır. u.optoelektronik uygulamalar için uygun

Some physical properties obtained for both half-Heusler compounds and binary semiconductors help us find new half-Heusler semiconductors favorable for optoelectronic applications. In this work, we examine the half-Heusler compound, NaYSi, which has industrial applications, using density-functional theory calculations.

This fact motivated us to investigate the electronic, elastic and dynamic properties of NaYSi ternary compound. Also, until now the electronic structures and mechanical properties of NaYSi compound has been almost uninvestigated. Therefore, the aim of this work is to give a detailed 
description of the behavior of elastic, electronic, vibrational and optic of NaYSi compound by using first principle calculations.

\section{Material and Methods}

VASP based on density functional theory (DFT) is used in all calculations [10-13]. The interaction between electron and ion is described by using the Projector Augmented Wave (PAW) method. The exchange and correlation potentials are described by the generalized gradient approximation (GGA) of Perdew and Zunger-type functional [14-15]. 700 $\mathrm{eV}$ cut-off energy and $15 \times 15 \times 15 \mathrm{k}$-points have been used in the Brillouin zone. The total energies converged to less than $10^{-5} \mathrm{ev} /$ atom.

\section{Results and/or Discussion}

\subsection{Structural Properties}

The half-Heusler NaYSi compound has cubic MgAgAs structure with space group F43m (216). As shown in Figure 1, the atomic positions of $\mathrm{Na}, \mathrm{Y}$ and $\mathrm{Si}$ atoms are $(0.5 ; 0.5 ; 0.5),(0.25$; $0.25 ; 0.25)$ and $\mathrm{Si}(0 ; 0 ; 0)$, respectively. In the equilibrium geometry calculation, all atomic positions and volume of the unit cell were relaxed (ISIF=3 in VASP). Bulk modulus (B) was determined the Murnaghan's equation of state E (V) curves. The results obtained for the compound are given in Table 1 comparatively [16].

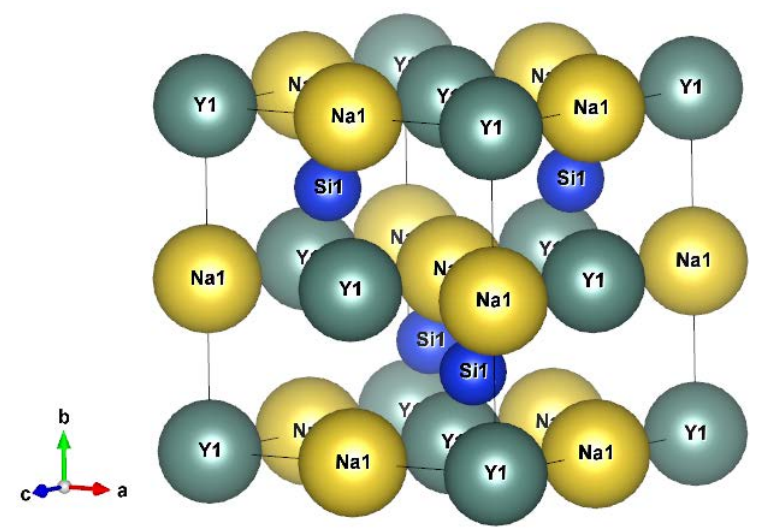

Fig. 1. The unit cell of the NaYSi.

Table 1. Calculated lattice. parameters $a_{0}(\AA)$, cell volume $V_{0}\left(\AA^{3}\right)$, bulk modulus (GPa) , band gap $\mathbf{E}_{g}$ and other calculated results (a Ref [16]). 


\begin{tabular}{ccccc}
\hline Reference & $\boldsymbol{a}_{\boldsymbol{o}}(\AA)$ & $\boldsymbol{V}_{\boldsymbol{o}}\left(\AA^{3}\right)$ & $\mathrm{B}(\mathrm{GPa})$ & $\mathrm{E}_{\boldsymbol{g}}$ \\
\hline Present & 6.873 & 80.53 & 44.5 & 3.6 \\
PBE-GGA & 6.870 & & & \\
\hline
\end{tabular}

\subsection{Electronic Properties}

We have computed the electronic band structure as depicted in Figure 2. The results indicate that NaYSi has direct band transition (X-X) with band gap $3.6 \mathrm{eV}$.

Figure 3 gives the total and partial density of states. In the conduction band, the largest contribution comes from the $\mathrm{Y} d$-states above the Fermi energy level, although the $p$-states with the Na $s$ - and Si $p$ - states also make small contributions to total density of the states. The next bands below the Fermi level orginate from the hybridization between Y $d$-states and Si $p$-states. On the other hand, Si s-states are in the lowest energy region of the valence band.

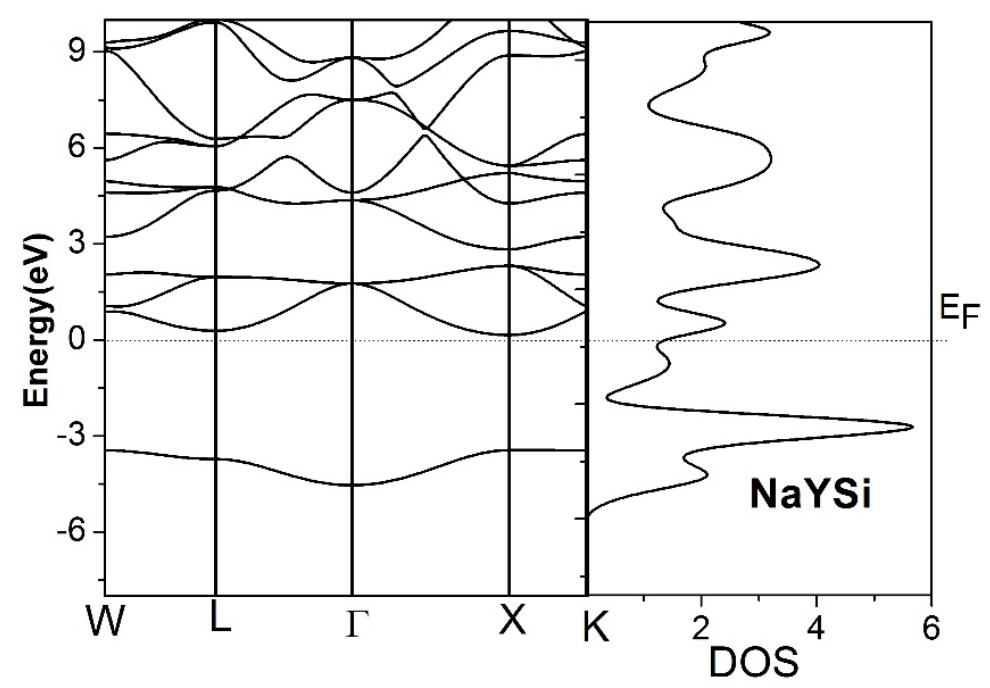

Fig. 2 Calculated electronic band structure for NaYSi. 


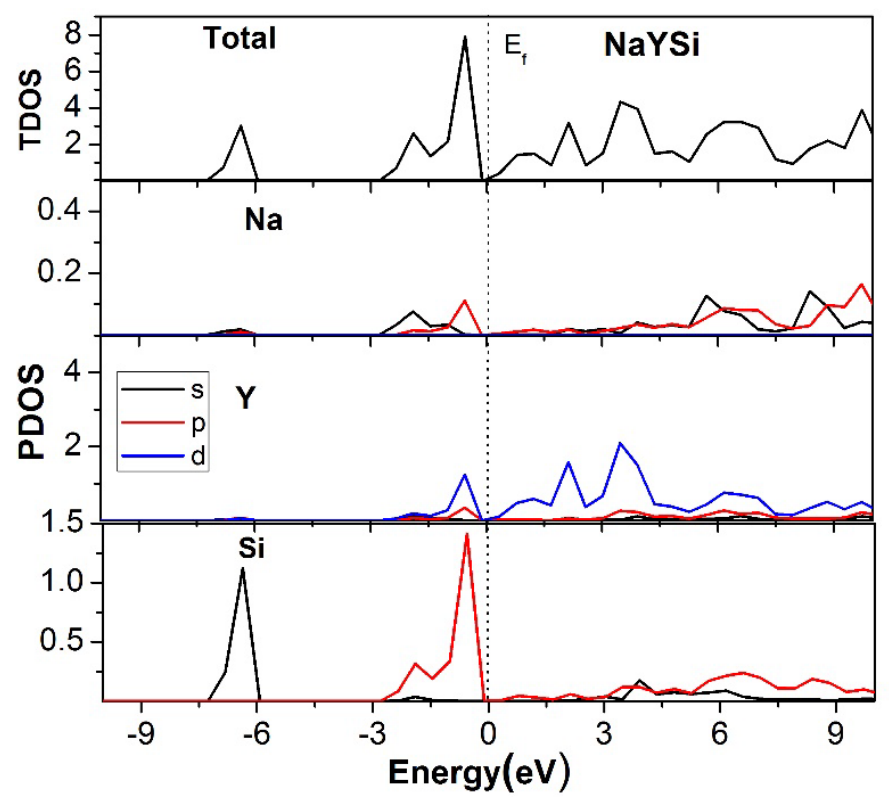

Fig. 3 Calculated partial dos (TDOS) and for NaYSi.

\subsection{Elastic Properties}

Elastic constants provide important information in understanding the mechanical and dynamic behavior of crystals.

In particular, they provide information on the stiffness and stability of materials. As know the forces and the elastic constants are functions of the first-order and secondorder derivatives of the potentials. Hence, to study the stability of title compound in MgAgAs structure, we have calculated the elastic constants at equilibrium lattice parameter. The results are listed in Table 2.

The mechanical stabilities as a function of pressure $(\mathrm{P}=0)$ can be calculated by using the following equation:

$$
\frac{1}{3}\left(C_{11}+2 C_{12}+P\right)>0 ; \frac{1}{2}\left(C_{11}-C_{12}-2 P\right)>0 ;\left(C_{44}-P\right)>0
$$

Obviously, NaYSi compound is mechanically stable according to above mechanical stability conditions.

Zener anisotropy factor A is 1 value in isotropic crystals. The calculated A is 0.678 for this compound, which indicates that NaYSi is anisotropical material. The lower limit value of poisson's ratio ( $v$ ) for central forces in solids and ionic crystals is 0.25 and the upper limit value is 0.5 [17]. In 
this study, interatomic forces in NaYSi have central forces and ionic bonding due to Pugy ratio $(v=0.281)$.

$\mathrm{C}_{12}-\mathrm{C}_{44}$ is Cauchy's pressure, give the ductile or brittle behaviour of materials. Compounds with a positive Cauchy pressure exhibit mechanic behaviour (ductile) [18]. As seen in the Table 2, the investigated compound has a positive Cauchy pressure and thus the ductile behaviour. The critical value is the G/B ratio, if the ratio is less than 0.57, the material will behave ductile if not brittle [19]. The calculated value of the G/B ratio is 0.512 , indicating that this compound has a ductile manner (Table 2).

Table 2. Elastic constants $C_{\mathrm{ij}}(\mathrm{GPa})$, bulk modulus $B(\mathrm{GPa})$, shear modulus $G(\mathrm{GPa})$, Young Modulus E (GPa), zener Anisotropy factor $(A)$, poisson's ratio $(U), B / G$ ratio and Cauchy's pressure of NaYSi.

\begin{tabular}{lcccccccccc}
\hline $\mathbf{P}$ & $\mathbf{C}_{\mathbf{1 1}}$ & $\mathbf{C}_{\mathbf{1 2}}$ & $\mathbf{C}_{\mathbf{4 4}}$ & $\mathbf{B}$ & $\mathbf{G}$ & $\mathbf{E}$ & $\mathbf{A}$ & $\mathbf{v}$ & $\mathbf{G} / \mathbf{B}$ & $\mathbf{C}_{\mathbf{1 2}}-\mathbf{C}_{\mathbf{4 4}}$ \\
(Gpa) & & & & & & & & & & \\
\hline This study & 83.1 & 25.3 & 19.6 & 44.5 & 22.8 & 58.4 & 0.678 & 0.281 & 0.512 & 5.7 \\
Theory [20] & 83.0 & 28.0 & 23.0 & & & & & & & \\
\hline
\end{tabular}

\subsection{Vibrational Properties}

To investigate the vibrational properties, the phonon spectra of the title compound were computed using the PHONOPY code [21]. The phonon dispersion graph has been plotted using $2 \times 2 \times 2$ supercell with 32 atoms.

As shown in Fig. 4, phonon distribution curves are given with several high symmetry directions. Since this compound has not been studied up to now, it is important to describe their vibrational properties. Since this compound has three atoms in the primitive cell, it has nine phonon branches, including three acoustic and six optical branches. In Fig. 4, none of the branches involves a soft mode in the Brillouin zone, indicating that the compound is dynamically stable. 


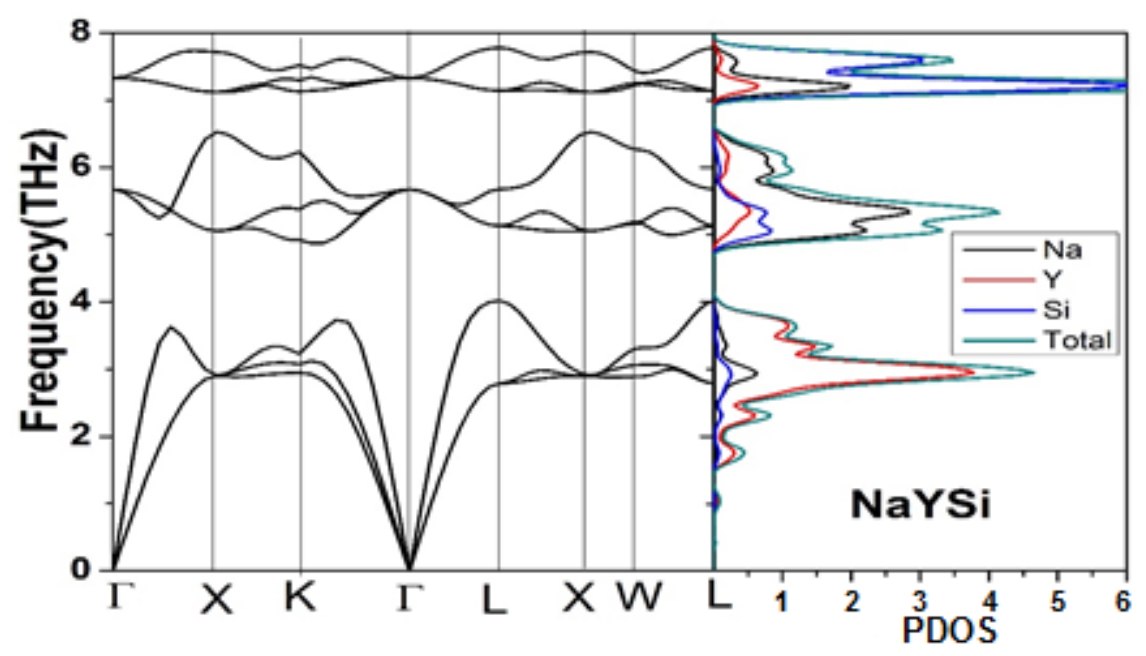

Fig. 4 Phonon dispersion curves for NaYSi.

\subsection{Optical Properties}

The optical properties have been investigated in order to more comprehensively understand the electronic structure of materials. The optical properties of material are defined by the complex dielectric function $\varepsilon(\omega)$, which governs the propagation behavior of radiation in a medium. Optical parameterizations, extinction coefficient $\mathrm{k}(\omega)$, the energy loss function $\mathrm{L}(\omega)$, the refractive index $n(\omega)$ and reflectivity $R(\omega)$, were created using the Kramers-Kroning equation. Calculated results for dielectric functions and $n(\omega)$ and $L(\omega)$ are summarized in Figure 5 (a-f), up to $30 \mathrm{eV}$, respectively.

The energy loss of a fast electron traversing is describe by the loss energy function $\mathrm{L}(\omega)$. The highest energy loss function occurs in the ultraviolet region as seen Figure 5(d). This peak value is located at $12 \mathrm{eV}$. 

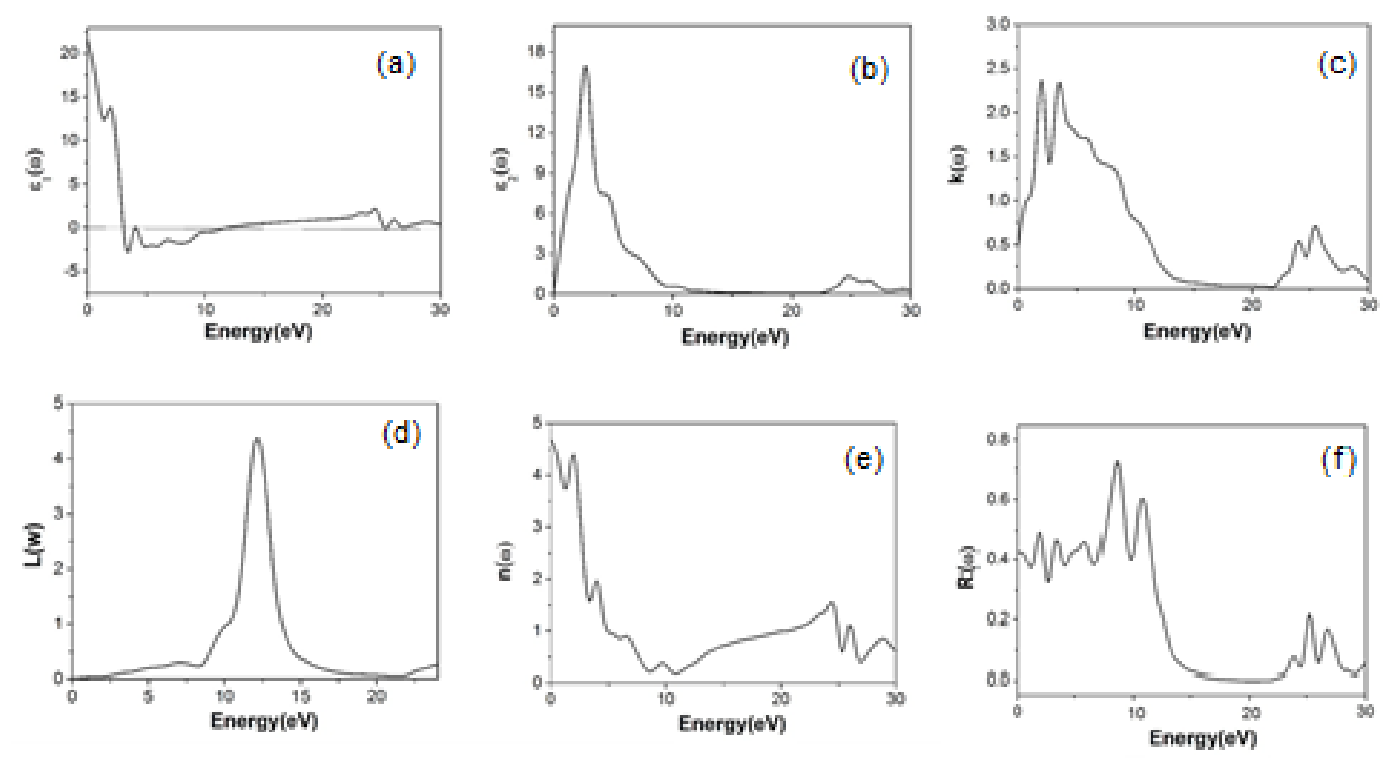

Fig 5. Optical properties for NaYSi.

\section{Conclusion}

We have investigated the elastic, electronic, vibrational and optic of NaYSi compound by using first principle calculations. A direct band gap of $3.6 \mathrm{eV}$ was observed in the calculated electronic structure profile and showed compound semiconductor behavior. This compound exhibited mechanical stability in the cubic phase from the calculated elastic constants. It is also an anisotropic material. Considering the positive phonon frequencies, it has been observed that a dynamic stability. Further the optical properties analysed for the energy range of 0-40 eV. These calculations provide promising information for the compound's use in optoelectronic applications.

\section{References}

[1] Nowotny, H., \& Bachmayer, K. (1950). Die Verbindungen LiMgP, LiZnP und LiZnAs. Monatshefte für Chemie und verwandte Teile anderer Wissenschaften, 81(4), 488-496.

[2] Kandpal, H. C., Felser, C., \& Seshadri, R. (2006). Covalent bonding and the nature of band gaps in some half-Heusler compounds. Journal of Physics D: Applied Physics, 39(5), 776. 
[3] Mehnane, H., Bekkouche, B., Kacimi, S., Hallouche, A., Djermouni, M., \& Zaoui, A. (2012). First-principles study of new half Heusler for optoelectronic applications. Superlattices and Microstructures, 51(6), 772-784.

[4] Curtarolo, S., Hart, G. L., Nardelli, M. B., Mingo, N., Sanvito, S., \& Levy, O. (2013). The highthroughput highway to computational materials design. Nature materials, 12(3), 191-201.

[5] Öğüt, S., \& Rabe, K. M. (1995). Band gap and stability in the ternary intermetallic compounds NiSnM (M= Ti, Zr, Hf): A first-principles study. Physical Review B, 51(16), 10443.

[6] Kieven, D., Klenk, R., Naghavi, S., Felser, C., \& Gruhn, T. (2010). I-II-V half-Heusler compounds for optoelectronics: Ab initio calculations. Physical Review B, 81(7), 075208.

[7] Gruhn, T. (2010). Comparative ab initio study of half-Heusler compounds for optoelectronic applications. Physical Review B, 82(12), 125210.

[8] Casper, F., Seshadri, R., \& Felser, C. (2009). Semiconducting half-Heusler and LiGaGe structure type compounds. physica status solidi (a), 206(5), 1090-1095.

[9] Roy, A., Bennett, J. W., Rabe, K. M., \& Vanderbilt, D. (2012). Half-Heusler semiconductors as piezoelectrics. Physical review letters, 109(3), 037602.

[10] Kresse, G., \& Hafner, J. (1993). Ab initio molecular dynamics for liquid metals. Physical review $B, 47(1), 558$.

[11] Kresse, G., \& Hafner, J. (1994). Ab initio molecular-dynamics simulation of the liquidmetal-amorphous-semiconductor transition in germanium. Physical Review B, 49(20), 14251.

[12] Kresse, G., \& Furthmüller, J. (1996). Efficiency of ab-initio total energy calculations for metals and semiconductors using a plane-wave basis set. Computational materials science, 6(1), 15-50.

[13] Kresse, G., \& Furthmüller, J. (1996). Efficient iterative schemes for ab initio total-energy calculations using a plane-wave basis set. Physical review B, 54(16), 11169.

[14] Perdew, J. P., Burke, K., \& Ernzerhof, M. (1996). Generalized gradient approximation made simple. Physical review letters, 77(18), 3865.

[15] Perdew, J. P., Burke, K., \& Ernzerhof, M. (1997). Generalized gradient approximation made simple (vol 77, pg 3865, 1996). Physical review letters, 78(7), 1396-1396.

[16] Kacimi, S., Mehnane, H., \& Zaoui, A. (2014). I-II-V and I-III-IV half-Heusler compounds for optoelectronic applications: Comparative $\mathrm{ab}$ initio study. Journal of Alloys and Compounds, 587, 451-458. 
[17] Fu, H., Li, D., Peng, F., Gao, T., \& Cheng, X. (2008). Ab initio calculations of elastic constants and thermodynamic properties of NiAl under high pressures. Computational Materials Science, 44(2), 774-778.

[18] Pettifor, D. G. (1992). Theoretical predictions of structure and related properties of intermetallics. Materials science and technology, 8(4), 345-349.

[19] Ciftci, Y. O., \& Evecen, M. (2018). First principle study of structural, electronic, mechanical, dynamic and optical properties of half-Heusler compound LiScSi under pressure. Phase Transitions, 91(12), 1206-1222.

[20] Roy, A. (2011). First-principles study of electromechanical and polar properties in perovskite oxides and half-Heusler semiconductors. Rutgers The State University of New JerseyNewBrunswick.

[21] Togo, A. (2012). Phonopy Manual Release 1.6. 2. 